\title{
Separation of memory span and learning rate: evidence from behavior and spontaneous brain activity in older adults
}

\section{Shuyuan Chen}

Institute of Psychology Chinese Academy of Sciences

\section{Rui Li}

Institute of Psychology Chinese Academy of Sciences

\section{Pengyun Wang}

Institute of Psychology Chinese Academy of Sciences

Juan Li ( $D$ lijuan@psych.ac.cn )

Chinese Academy of Sciences https://orcid.org/0000-0003-2322-515X

\section{Research}

Keywords: memory span, learning rate, separation, aging, spontaneous brain activity

Posted Date: September 17th, 2020

DOI: https://doi.org/10.21203/rs.3.rs-78186/v1

License: (c) (i) This work is licensed under a Creative Commons Attribution 4.0 International License. Read Full License 


\section{Abstract}

Objective: It is unclear how the ability to initially acquire information in a first learning trial relates to learning rate in subsequent repeated trials. The separation of memory span and learning rate is an important psychological dilemma that remains unaddressed. Given the potential effects of aging on memory and learning, this study investigated behavior and spontaneous brain activity in adults in the context of memory span and learning rate.

Method: We enrolled a total of 758 participants, including 707 healthy older adults and 51 mild cognitive impairment $(\mathrm{MCl})$ patients. Sixty-five participants out of 707 completed resting-state functional magnetic resonance imaging (fMRI) scanning.

Results: Behaviorally, memory span and learning rate were not correlated with each other in the pairedassociative learning test (PALT) but were negatively correlated in the auditory verbal learning test (AVLT). This indicated that the relationship between memory span and learning rate for item memory might be differentially affected by aging. Interaction analysis confirmed that these two capacities were differentially affected by test type (associative memory vs. item memory). Additionally, at the level of brain activity, distinct directions of correlation coefficients and brain regions for memory span and learning rate were detected using correlation analysis between behavioral performance and spontaneous brain activity. Regarding pathological aging, none of the correlations between memory span and learning rate were significant in either PALT or AVLT in $\mathrm{MCl}$.

Conclusion: This study provides novel evidence for the dissociation of memory span and learning rate at behavioral and brain activity levels, which may have useful applications in detecting cognitive deficits or conducting cognitive interventions.

\section{Introduction}

There are distinguishing differences in individuals' ability to learn versus reserve information. Zerr et al. found that learners who can memorize information quickly keep information more durably in their brains (Zerr et al. 2018). Furthermore, individuals also vary in their memory span and learning rate, which are two distinguishable processes. As suggested by Lezak, memory span (or memory capacity) refers to the capacity of an individual to learn information in a single trial. Learning rate reflects increments in learning, that is, the ability to encode new information while retrieving old information that has been memorized in a previous trial, and to integrate inter-trial information. This can be deduced from the slope of the learning curve across multiple learning trials (Lezak 2004).

The relationship between learning rate and memory span is unclear. That is, we do not yet know how the ability to initially acquire information in the first trial relates to increments in learning in subsequent trials or whether people who acquire more information in the first trial are also quick learners. This is plausible but does not seem to be the case. Zerr et al. (2018) reported that memory span and learning rate relied on different cognitive abilities in younger adults (IQ and processing-speed, respectively), although these 
capacities were positively correlated with each other. These results indicate that memory span and learning rate may be two distinct processes. To our knowledge, no study has directly examined whether memory span and learning rate are two independent processes or are related, partly because they covary in healthy young adults as two sub-functions of memory. The covariation of memory span and learning rate restricts the range in variation of performance in young adults, making it difficult to observe individual differences.

Learning rate is vulnerable to age-related memory deficits and brain injury (Almond et al. 2013). Thus, it is more feasible to assess functional separation between memory span and learning rate in older adults, even in individuals with mild cognitive impairment $(\mathrm{MCl})$. Older adults are generally memory impaired in the first trial and learning rate in multi-trial tests compared to younger adults (Davis et al. 2003; Dunlosky \& Salthouse 1996; Kramer et al. 2003). For example, older adults recall fewer items in a single trial and learn at a lower rate in multi-trial learning tests than do younger individuals (all participants' age range from 18 to 94) (Dunlosky \& Salthouse 1996). $\mathrm{MCl}$ is considered a transitional stage between normal aging and diagnosis of clinical dementia (Petersen 2004). MCI patients are enrolled in this study because they are disproportionately deficited in some subtypes of memory. For example, in contrast to their deficits in recollection, $\mathrm{MCl}$ patients had relatively intact familiarity (Anderson et al. 2008; Serra et al. 2010; Westerberg et al. 2006; Wolk et al. 2008). Recollection and familiarity are two independent processes during memory retrieval (Yonelinas 2002). For another example, it has been well established that associative memory has been found disproportionately impaired in contrast to item memory in $\mathrm{MCl}$ patients (Hanseeuw et al. 2011; Stella et al. 2009). Learning rate is negatively affected in MCl patients. Compared to cognitively normal older adults, the learning rate in amnestic $\mathrm{MCl}$ patients is lower (Moulin et al. 2004). In addition, episodic memory tests with multiple learning-test trials are sensitive in detecting $\mathrm{MCl}$ patients (Moulin et al. 2004). However, whether memory span and learning rate in healthy older adults and $\mathrm{MCl}$ patients are disproportionally or correlate with each other impaired remains unknown.

Neuropsychological tests, such as the auditory verbal learning test (AVLT) and the paired-associative learning test (PALT), are commonly used in clinical practice to assess episodic memory in normal older adults and patients with neurodegenerative disorders including $\mathrm{MCl}$, affecting the slope of the curve of learning (Machts et al. 2014). We hypothesize that item memory (the ability to memorize a single item) and associative memory (the capacity to remember item-item and item-context relationships) might have disparate effects on memory span relative to learning rate (Ranganath 2015). In the present study, we used PALT as an indicator of associative memory and AVLT as an indicator of item memory. We calculated memory span and learning rate in both PALT and AVLT.

At the level of neural activity, we aimed to determine whether memory span and learning rate were related to the different levels of spontaneous resting-state brain activity. We explored this issue systematically from three progressive indexes: single-voxel level (amplitude of low-frequency fluctuation, ALFF), regional brain network level (regional homogeneity, ReHo), and whole-brain network level (degree of centrality, DC). To our knowledge, there has not been any studies that studied the relationship of ALFF, ReHo, and DC and cognition in healthy older adults. Whereas abnormal ALFF, ReHo, and DC are important indexes of 
neurodegenerative disease. It has been suggested that the ALFF may indicate regional spontaneous neuronal activity (Yong et al. 2004; Yu-Feng et al. 2007). Pan et al. found that, relative to healthy controls, amnestic $\mathrm{MCl}$ patients showed decreased ALFFs in the bilateral precuneus/posterior cingulate cortices, left occipitotemporal cortex, left middle occipital gyrus, and left inferior temporal gyrus in a meta-analysis of resting-state fMRI study (Pan et al. 2017). In addition, a meta-regression analysis demonstrated that the increased severity of cognitive impairment in aMCl patients was associated with greater decreases in ALFFs in the cuneus/precuneus cortices (Pan et al. 2017). Abnormal ReHo has been observed in schizophrenia (Liu et al. 2013) and Alzheimer's disease (AD) (Yong et al. 2007) in the resting-state fMRI. Yong et al. (Yong et al. 2007) found that in some regions showing AD-related changes in ReHo, such as the posterior cingulate cortex, the amplitude of low frequency fluctuations (ALFF) was decreased. In $\mathrm{aMCl} / \mathrm{MCl}$, the higher ReHo was found in the left inferior parietal lobule, left posterior cingulate cortex/precuneus (PCC/PCu), left inferior temporal gyrus (ITG) (Yuan et al. 2016; Zhang et al. 2012). Furthermore, in the aMCl patients, there is a positive correlation between the ReHo index in the left PCC with the AVLT-Immediate Recall scores (Yuan et al. 2016), whereas there is a negative correlation between the ReHo index in the left inferior parietal lobule (IPL) with the AVLT-Immediate and delayed Recall scores (Zhang et al. 2012). For DC, Guo et al. found that AD patients with depression had lower DC values in the right middle frontal, precentral, and postcentral gyrus than non-AD patients with depression. Furthermore, seed-based analysis revealed decreased connectivity between the precentral and postcentral gyrus to the supplementary motor area and middle cingulum (Guo et al. 2016). In conclusion, ALFF and ReHo have association with the precuneus and left inferior temporal gyrus, also with ALFF, ReHo and DC in cingulate cortices.

In summary, the purpose of the present study was to examine whether memory span and learning rate were two independent mnemonic processes at the level of (1) behavior and (2) spontaneous brain activity in (3) healthy older adults and those diagnosed with $\mathrm{MCl}$.

\section{Materials And Methods}

\section{Participants}

All participants were from a community-based $\mathrm{MCl}$ screening project (Yu et al. 2012). To meet eligibility criteria, individuals were required to be ${ }^{3} 57$ years old. Experienced psychiatrists performed all clinical diagnoses. Older adult participants with normal cognition ability had to meet the following criteria: (i) report right-handedness, (ii) score $\geq 22$ on the Montreal Cognitive Assessment-Beijing Version (MoCABeijing) (Yu et al. 2012) or score $\geq 25$ in the mini-mental state examination (MMSE), (iii) have a global Clinical Dementia Rating (CDR) score of 0, (iv) have normal capabilities of activities of daily living (ADL) (Lawton \& Brody 1969).

$\mathrm{MCl}$ patients had to meet the following criteria: (i) report right-handedness, (ii) have subjective complaints of memory loss, preferably corroborated by an informant, (iii) have MMSE score $\geq 24$ for individuals with more than 6 years of education and $\geq 20$ for individuals with $£ 6$ years of education, (iv) have a global 
CDR score of 0.5 with a memory score of 0.5 or 1 , (v) have normal capabilities of ADL (Lawton \& Brody 1969), and (vi) have no dementia based on the Diagnostic and Statistical Manual of Mental Disorders, Fourth Edition (DSM-IV). Participants were excluded from the study if they had (i) a history of head trauma resulting in loss of consciousness for more than $1 \mathrm{~h}$, (ii) alcohol or substance abuse, or (iii) current psychiatric disorders and medications known to affect the central nervous system.

In total, 758 participants were enrolled in our study, including 707 healthy older adults (331 men, 376 women) and $51 \mathrm{MCl}$ patients (23 men, 28 women). Out of the 707 healthy older adults, 642 healthy participants and the $51 \mathrm{MCl}$ patients completed the Neuropsychological Test Battery (NTB) (Seidman 1998), including the Digit Span (forward and backward), Category Fluency Test (CFT), Block Design, Picture Completion, Visual Discrimination test (functional connections, semantic connections, recognition, visual matching, and reasoning) (Wechsler 1984), paired-associative memory, and auditory verbal learning test. The remaining 69 participants of the 707 healthy older adults only completed the paired-associative memory and auditory verbal learning test and underwent resting-state fMRI scanning after completing the NTB within a month. NTB was used to examine whether memory span and learning rate were related to different levels of spontaneous brain activity in resting-state. The demographic characteristics of the participants are presented in Table 1.

Table 1 Demographic characteristics and neuropsychological measures of the participants (mean \pm s.d.)

\begin{tabular}{ccccc}
\hline Characteristics & Healthy older adults $(\mathrm{n}=707)$ & MCI $(\mathrm{n}=51)$ & $t / \chi 2$ & $p$ \\
\hline Age(years) & $69.29(6.37)$ & $69.67(6.67)$ & 0.40 & 0.69 \\
Gender(F/M) & $331 / 376$ & $23 / 28$ & 0.06 & 0.89 \\
Education(years) & $11.95(4.42)$ & $11.10(4.67)$ & 1.33 & 0.18 \\
MMSE & $28.03(1.51)(\mathrm{n}=642)$ & $27.37(2.47)$ & 1.87 & 0.07 \\
Digit span (forward) & $10.00(2.01)(\mathrm{n}=642)$ & $9.75(2.19)$ & 0.86 & 0.39 \\
Digit span (backward) & $5.93(2.06)(\mathrm{n}=642)$ & $5.90(2.68)$ & 0.07 & 0.95 \\
Category Fluency Test & $11.18(3.01)(\mathrm{n}=642)$ & $10.61(3.18)$ & 1.28 & 0.20 \\
Picture Completion & $10.24(3.50)(\mathrm{n}=642)$ & $11.47(12.93)$ & -.0 .68 & 0.50 \\
Block design & $28.63(8.25)(\mathrm{n}=642)$ & $26.86(8.41)$ & 1.47 & 0.14 \\
Visual Discrimination test: & & & & \\
Functional association & $3.68(0.59)$ & $3.53(0.76)$ & 1.38 & 0.17 \\
Semantic association & $3.28(0.81)$ & $3.25(0.77)$ & 0.18 & 0.86 \\
recognition & $5.91(1.41)$ & $5.79(1.55)$ & 0.82 & 0.41 \\
Visual matching and reasoning & $5.38(2.08)$ & $4.41(2.55)$ & 2.91 & $<0.01$ \\
PALT - MS1 & $0.17(0.12)$ & $0.14(0.10)$ & 1.84 & 0.07 \\
AVLT - MS1 & $0.38(0.15)$ & $0.34(0.14)$ & 1.89 & 0.06 \\
PALT - LR2 & $0.24(0.15)$ & $0.25(0.16)$ & -0.43 & 0.67 \\
AVLT - LR2 & $0.28(0.16)$ & $0.28(0.17)$ & 0.28 & 0.78 \\
\hline
\end{tabular}

Note. MoCA, Montreal cognitive assessment. PALT, paired-associative learning test. AVLT, auditory verbal learning test. MS, memory span. LR, learning rate. 1, 2, Corrected item and associative recall Memory span: PALT, trial1/9; AVLT, trial1/15 Learning rate: PALT, (trial 3-trial1)/9; AVLT, (trial 3-trial1)/15.

\section{Paired-associative learning test (PALT)}

The PALT (Xu \& Wu 1986) was used to assess participants' associative memory ability. First, the participants learned 12 word-pairs consisting of six novel associations (e.g., teacher-railway) and six semantically related associations (e.g., sun-moon). All 12 word-pairs were presented audibly at a rate of 3 
$s$ per word pair, with intervals of $2 \mathrm{~s}$ between pairs. After the study session, participants were asked to complete a cued recall task in which the first word of the pair was provided, in which they had to recall the other paired word within $5 \mathrm{~s}$. A correctly recalled word was scored 0.5 for semantically related associations and 1 for novel associations. The procedure was repeated three times with different word pair orders.

\section{Auditory verbal learning test (AVLT)}

AVLT (WHO-UCLA version) (Maj et al. 1994) consists of five learning trials. A list of 15 words was read aloud at a rate of approximately one word per second. Participants were asked to immediately recall as many items as possible. This study-test trial was repeated five times. Participants were asked to recall the words 20 min after the fifth trial (first delayed recall). After the seventh trial of 15 distractor words, subjects were then asked to remember as many objects on the list after $30 \mathrm{~min}$ in any order (second delayed recall). Only the first three study-test trials were analyzed such that the number of trials for the PALT and AVLT was equal.

\section{Functional magnetic resonance imaging (fMRI) image acquisition}

A 3-Tesla Siemens Trio scanner (Erlangen, Germany) equipped for echo planar imaging (EPI) at the Beijing MRI Center for Brain Research was used for image acquisition. During the scan, participants laid in a supine orientation with their head snugly fixed by a belt and foam pads to minimize head motion. Participants were instructed to lie quietly, keep their eyes closed, and not to think of anything in particular. For each participant, 200 functional images were obtained using the following parameters: time repetition $(T R)=2,000 \mathrm{~ms}$, time echo $(T E)=30 \mathrm{~ms}$, flip angle $=90^{\circ}$, field of view $(F O V)=200 \times 200 \mathrm{~mm}^{2}, 33$ axial slices, thickness $=3.0 \mathrm{~mm}$, gap $=0.6 \mathrm{~mm}$, acquisition matrix $=64 \times 64$, and in-plane resolution $=3.125 \times$ 3.125. A high-resolution 3-D T1-weighted structural image was acquired for each participant using a magnetization-prepared rapid gradient echo (MPRAGE) sequence: 176 slices, acquisition matrix $=256 \times$ 256 , voxel size $\times 1 \times 1 \times 1 \mathrm{~mm}^{3}, \mathrm{TR}=1,900 \mathrm{~ms}, \mathrm{TE}=2.2 \mathrm{~ms}$, and flip angle $=9^{\circ}$.

\section{Image Processing}

Resting-state fMRI data analyses were processed with Statistical Parametric Mapping (SPM8, http://www.fil.ion.ucl.ac.uk/spm) and toolbox for Data Processing and Analysis of Brain Imaging DPABIV2.3 (Yan et al. 2016a).

Preprocessing: data preprocessing included removing the first 10 time points, slice timing correction, and within-subject spatial realignment. All included participants had head motion less than $3 \mathrm{~mm}$ in any one direction during scanning. Coregistration, segmentation, and writing normalization were performed using the unified segmentation of each participant's T1 image. Nuisance covariates, including head motion parameters, white matter signal, and cerebral spinal fluid signal were regressed out.

\section{ALFF analysis}


As an index of brain activity in single voxel level, ALFF is calculated in two steps. First, for a given voxel, a fast Fourier transform was used to convert the filtered time series to a frequency domain to obtain the power spectrum. Second, the power spectrum was then square-rooted and averaged across $0.01-0.1 \mathrm{~Hz}$ at each voxel (Yan et al. 2016b). ALFF is directly correlated with the intensity of spontaneous neural activity in the resting state with regards to energy metabolism (Qi et al. 2012). We have also conducted a between-subject spatial normalization to the Montreal Neurological Institute (MNI) coordinate space with $3 \times 3 \times 3 \mathrm{~mm}^{3}$ resampling and spatial smoothing with a 4-mm Gaussian kernel.

\section{ReHo analysis}

We calculated ReHo as the Kendall's coefficient of concordance (KCC) of a given voxel with its 26 most proximal voxels as a cluster. We then divided the KCC of a given voxel by the average KCC of the whole brain to reduce the influence of individual variations in the KCC value (Zang et al. 2004). Next, the standardized ReHo maps were spatially normalized to the MNI template and resampled at a resolution of $3 \times 3 \times 3 \mathrm{~mm}^{3}$ using the normalization parameters estimated during unified segmentation. Finally, we smoothed with a Gaussian kernel of $4 \mathrm{~mm}$ FWHM to reduce noise. High ReHo values indicate high synchronized oscillation of neurons in a given brain region (Zang et al. 2004).

\section{Degree of centrality}

DC was calculated according to the methods used in a previous study (Zhou et al. 2014). A correlation matrix was first obtained by computing Pearson correlation coefficients between the time courses of one voxel with all other voxel time courses within a predefined grey matter mask. This grey matter template was released as part of the prior tissue in SPM8 that included tissue with grey matter probabilities $>20 \%$. An undirected adjacency matrix was then obtained by thresholding each correlation at $r>0.25$ to remove weak correlations caused by noise. Next, weighted DC was computed as the sum of the connections between a given voxel and all other voxels. Finally, the individual-level voxel-wise DC was converted into a $z$-score map. The resulting maps were then registered into MNI space with $3 \mathrm{~mm}^{3}$ cubic voxels using the transformation information acquired from DARTEL. A smooth kernel of $4 \mathrm{~mm}$ was applied after registration. DC reflects how much a node influences area throughout the entire brain. ALFF, ReHo, and DC have high test-retest reliability (Li et al. 2012; Wang et al. 2017; Zuo et al. 2010).

\section{Statistical analysis of neuropsychological data}

As item and associative memory tests have different full scores, we tested for differences in memory span and learning rate in corrected item and associative scores: Item memory (memory span =the number of words correctly recollected at trial 1divided by the full scores 15 of AVLT; Learning rate $=$ the difference between trial 3 and trial 1 ) divided by the full scores 15; associative memory (memory span= the number of words correctly recollected at trial 1divided by the full scores 9 of PALT; Learning rate $=$ the difference between trial 3 and trial 1) divided by the full scores 9). To evaluate the separation between memory span and learning rate, we verified the purpose through three different behavioral analysis 
methods. Pearson correlation analyses between memory span and learning rate corrected scores in PALT and AVLT were performed separately.

To examine whether the separation between memory span and learning rate was affected by memory type (associative memory vs. item memory, i.e. PALT vs. AVLT) in a different manner, repeated measures ANOVA was conducted on memory type (within-subject factor: PALT vs. AVLT) and cognitive processes (within-subject factor: memory span vs. learning rate) for the normal groups.

A forward stepwise regression was conducted for memory span and learning rate based on relevant cognitive batteries, with the goal to assess the relative contributions of three predictor variables processing speed (verbal fluency), memory ability (as assessed by digit span), and intellectual ability (as assessed by picture completion, block design, visual discrimination test, and Category Fluency Test) to two dependent variables memory span and learning rate.

To verify whether the difference (dependent variable) between memory span and learning rate was modulated by age and education (predictor variables), a two-block hierarchical linear regression model was conducted to determine the modulatory effects of age or education. For age, age and memory span were entered in the first block. In block 2, age, memory span, and the interaction term of age and memory span were added. If the $\mathrm{R}$ square change of the model or the interaction term of age and memory span were not significant, the difference between memory span and learning rate was not modulated by age.

The processed gray matter images were entered into a regression model using DPABI to measure correlations between brain activity (ALFF, ReHo, and DC) and scores on neuropsychological measures. Pearson correlation analyses between the brain activity indexes (ALFF, ReHo, and DC) and memory scores (memory span and learning rate) in PALT and AVLT using a grey mask were performed separately. According to Bonferroni correction, to test three independent hypotheses on the same data at 0.05 significance level, instead of using a $p$ value threshold of 0.05 , one would use a stricter threshold of $0.0167(0.05 / 3)$. In the present study, to control the effect of multiple comparisons, we have adopted a stricter standard for each brain imaging analysis: the false discovery rate (FDR) at $p<0.01$ (both voxel and cluster).

To further examine whether the relationship between memory span and learning rate on normal older adults still existed on $\mathrm{MCl}$ patients, repeated measures ANOVA was conducted with memory type (PALT vs. AVLT) and memory capability (memory span vs. learning rate) for $\mathrm{MCl}$ groups.

\section{Results}

\section{The separation of memory span and learning rate in behavioral performance for normal group}

To evaluate the relationship between memory span and learning rate, we first calculated the correlation between memory span and learning rate in both PALT and AVLT. For the normal group, there was no significant correlation between memory span and learning rate in PALT $(n=707, r=-0.030,[-0.103,0.043]$, 
$\left.p=0.432 ; \eta_{p}^{2}=0.001\right)$. In AVLT, memory span was negatively correlated with learning rate $(n=707, r=$ $\left.-0.268,[-0.339,-0.198], p<0.001 ; \eta_{p}^{2}=0.07\right)$. These results are shown in Fig. 1.

To investigate whether memory span and learning rate were differently affected by memory type (associative memory vs. item memory, i.e. PALT vs. AVLT), we conducted an ANOVA on memory type (PALT vs. AVLT) $\times$ memory capability (memory span vs. learning rate) for the normal and $\mathrm{MCl}$ groups. For the normal group, the interaction effect was significant $\left(F(1,706)=195.076 \rrbracket p<0.001 ; \eta_{p}{ }^{2}=0.216\right)$. Post hoc tests revealed that the memory span score was significantly higher than the learning rate score in $\operatorname{AVLT}\left(F(1,706)=112.140 \otimes p<0.001 ; \eta_{p}^{2}=0.137\right)$. In contrast, the memory span score was significantly lower than the learning rate score in $\operatorname{PALT}\left(F(1,706)=83.280 \otimes p<0.001 ; \eta_{p}{ }^{2}=0.105\right)$. Conversely, although both memory span $\left(F(1,706)=1134.320 \rrbracket p<0.001 ; \eta_{p}^{2}=0.616\right)$ and learning rate were higher in AVLT than in PALT $\left(F(1,706)=33.390 \otimes p<0.001 ; \eta_{p}{ }^{2}=0.045\right)$, the significant interaction effect indicated that the difference between AVLT and PALT in memory span was larger than that in learning rate; that is, memory span and learning rate were differently affected by memory type (PALT vs. AVLT) (Fig. 2).

As 69 of the 707 healthy older adults were used to examine whether memory span and learning rate were related to different levels of spontaneous brain activity in resting-state, we recalculated the correlation analysis as described above to ensure the representativeness of the 69 samples. Generally, the correlation pattern of the results for 69 samples was the same as that of all 707 participants. Nevertheless, the correlation between memory span and learning rate in PALT approached to marginal significance for 69 samples, the correlation between memory span and learning rate in PALT was not significant for 707 samples. Mainly, because the average score on the first trial of PALT was a little high for 69 samples. So to further improve the representativeness of 69 samples and to ensure the same data structure between PALT and AVLT, we removed 18 (data with scores 1 SD higher than the mean score on the first trial of PALT and AVLT) of the 69 healthy older adults from the dataset. The resulting pattern of the remaining 51 healthy older adults was more representative. Detailed descriptions are provided in the Supplementary Materials.

\section{Contribution of cognitive abilities to memory span and learning rate}

We combined scores for memory span and learning rate of PALT and AVLT separately to perform forward stepwise regression. We obtained two models for memory span, which are illustrated in Table 2.

According to Model 1, Category Fluency Test accounted for the full percentage of the explained variance. For Model 2, Category Fluency Test accounted for a greater percentage of the explained variance than did Picture Completion, among the variables. Table 3 depicts three models for learning rate. According to Model 1 and Model 2, the contribution of Picture Completion and Category Fluency Test to the percentages of the explained variance was opposite to memory span. Picture Completion mainly contributed to learning rate.

Table 2 Regression Results and Included cognitive abilities variables for memory span $(n=642)$ 


\begin{tabular}{ccccccc}
\hline 1 & test & $F$ & $R 2$ & $\Delta R 2$ & Standardized $\beta$ & Partial correlation $r$ \\
\hline 1 & & $112.177^{* * *}$ & 0.149 & 0.148 & & \\
& Category Fluency Test & & & & & \\
& & $75.353^{* * *}$ & 0.191 & 0.188 & & 0.386 \\
\multicolumn{2}{c}{ Category Fluency Test } & & & & 0.302 & 0.297 \\
\multicolumn{2}{c}{ Picture Completion } & & & & 0.221 & 0.204 \\
\hline
\end{tabular}

Table 3 Regression Results and Included cognitive abilities variables for Learning rate $(n=642)$

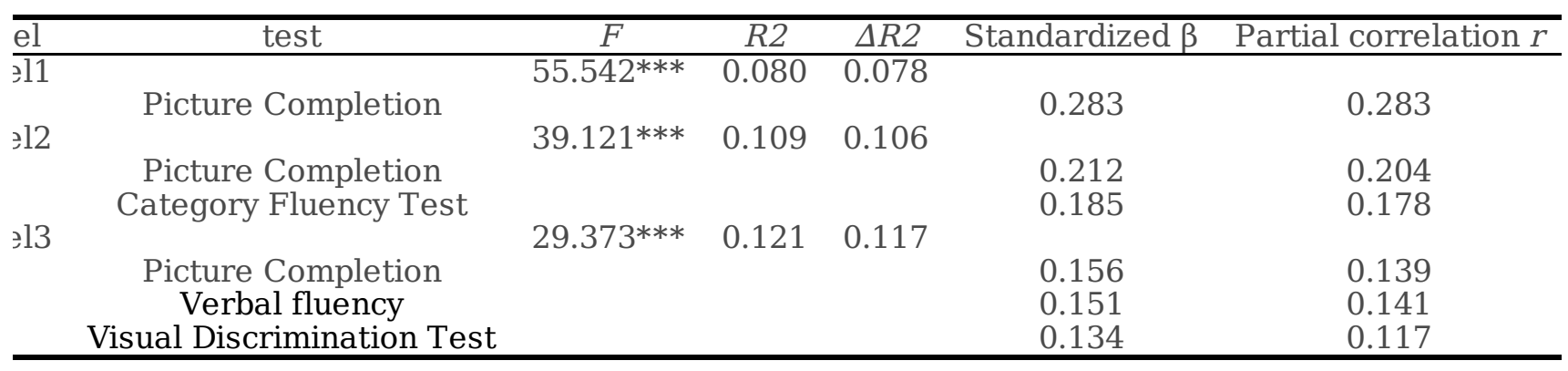

\section{Modulation of memory span and learning rate by age and education}

To examine whether differences between memory span and learning rate were modulated by age and education, we calculated the moderating effects of age and education. Neither of the moderating effects were significant for age (PALT, $\mathrm{R}^{2}=0.000, p=0.715 ; t=-0.365, p=0.715 ; \mathrm{AVLT}, \mathrm{R}^{2}=0.004, p=0.083 ; t=$ 1.734, $p=0.083$ ) or for education (PALT, $\mathrm{R}^{2}=0.004, p=0.073 ; t=-1.793, p=0.073 ; \mathrm{AVLT}^{2} \mathrm{R}^{2}=0.003, p=$ $0.117 ; t=1.567, p=0.117)$. These results indicated that the difference between memory span and learning rate was not related to age and education.

\section{Correlations between indexes of brain activity and behavioral performance}

As mentioned above, 51 healthy older adults who participated in fMRI scanning were selected for examination of whether memory span and learning rate were related to different levels of spontaneous brain activity in resting-state (table 4, supplementary material).

\section{Correlation between ALFF and behavioral performance}

In PALT, correlation analysis revealed that ALFF in the left amygdala (peak MNI coordinate: $-27,-3,-15$; number of voxels: 204) was positively correlated with learning rate. The correlation between memory span and ALFF in this region was not significant. We extracted the ALFF value of peak coordinates in this cluster to perform correlation analysis with PALT scores on memory span and learning rate separately. Further analysis revealed a robust difference between the two correlation coefficients (one-tailed Hotelling's $t$-test: $\left.t_{(48)}=7.881, p<0.001\right)$. No regions with a significant correlation between memory span and ALFF in PALT were detected, as shown in Fig. 3A-B.

In AVLT, one cluster in the right middle frontal gyrus (peak MNI coordinate: $36,-3,51$; number of voxels: 155) displayed a negative correlation between ALFF with memory span (depicted in Fig. 3C). We 
extracted the ALFF value of peak coordinates in this cluster to perform correlation analysis with memory span and learning rate in AVLT (Fig. 3D). Subsequent $t$-test revealed a significant difference between these two correlation coefficients (one-tailed Hotelling's $t$-test: $t_{(48)}=-2.540, p<0.01$ ). No brain region with a significant correlation between ALFF and learning rate in AVLT was detected.

\section{Correlation between ReHo and behavioral performance}

In PALT, we observed that the ReHo value of a region in the right inferior temporal gyrus (peak MNI coordinate: $60,-39,-21$, number of voxels: 251 ) was negatively correlated with memory span, while another region including parts of the left middle occipital gyrus and precuneus (peak MNI coordinate: -39, $-78,9$, number of voxels: 1083 ) was positively correlated with learning rate. We extracted the ReHo value of peak coordinates in the two clusters and performed correlation analysis between ReHo and PALT scores for memory span and learning rate. Subsequent $t$-test revealed that the correlation coefficient was larger for memory span than for learning rate in the right inferior temporal gyrus (one-tailed Hotelling's $t$ test: $t(48)=-3.231, p<0.01$ ). The correlation coefficient was smaller for memory span than for learning rate in the left middle occipital gyrus and precuneus (one-tailed Hotelling's $t$-test: $t(48)=4.352, p<0.01$ ).

In AVLT, correlation analysis revealed that the ReHo value of the left precuneus (peak MNI coordinate: 0 , $-60,45$; number of voxels: 197 ) was positively correlated to learning rate. We extracted the ReHo value of peak coordinates in this cluster to perform correlation analyses with AVLT scores on memory span and learning rate. Further analysis revealed a significant difference (one-tailed Hotelling's $t$-test: $t_{(48)}=3.214$, $p<0.01)$. We did not find any significant correlations between the ReHo values and memory span scores of AVLT. These results are shown in Fig. 4.

\section{Correlation between DC and behavioral performance}

In PALT, correlation analysis revealed that the DC value of the left precuneus and occipital lobe (peak MNI coordinate: -21, -90, 30; number of voxels: 134) was positively associated with learning rate across all 51 older adults. We extracted the DC value of peak coordinates of this region and performed correlation analyses with behavioral scores (memory span and learning rate). Subsequent $t$ test revealed a significant difference (one-tailed Hotelling's t-test: $t_{(48)}=2.428, p<0.01$ ).

In AVLT, the DC value of the region in the right precuneus (peak coordinate: $9,-69,45$; number of voxels: 161) was positively correlated with learning rate. We extracted the $D C$ value of peak coordinates of this region and performed correlation analyses with behavioral scores (memory span and learning rate).

Subsequent $t$-test revealed a significant difference between these two coefficients (one-tailed Hotelling's $t$ test: $t(48)=3.016, p<0.01)$. These results are shown in Fig. 5 .

\section{The separation of memory span and learning rate in behavioral performance for $\mathrm{MCl}$ groups}

For the $\mathrm{MCl}$ group, we observed a significant interaction between memory type and memory capability $\left(F(1,706)=19.487 \rrbracket p<0.001 ; \eta_{p}^{2}=0.027\right)$. ANOVA analysis confirmed a significant difference in memory 
span between PALT and $\operatorname{AVLT}\left(F(1,706)=79.530 \rrbracket p<0.001 ; \eta_{p}{ }^{2}=0.101\right)$, but no significant difference was observed in the learning rate between PALT and $\operatorname{AVLT}(p=0.278)$. We observed the same pattern as that in the normal group, whereby memory span score was significantly lower than learning rate score in PALT $\left(F(1,706)=18.260 \otimes p<0.001 ; \eta_{p}^{2}=0.025\right)$, however, memory span showed a trend being higher than learning rate in $\operatorname{AVLT}(p=0.055)$. These results implied that memory span and learning rate were affected by memory type (associative memory or item memory) in a distinct manner (see Fig. 1).

The correlation between memory span and learning rate was not significant in either PALT $(n=51, r=$ 0.089 , [0.015, 0.163], $\left.p=0.536 ; \eta_{p}{ }^{2}=0.008\right)$ or $\operatorname{AVLT}\left(n=51, r=-0.132,[-0.205,0.059], p=0.355 ; \eta_{p}{ }^{2}=\right.$ 0.008) (see Fig. 2).

\section{Discussion}

Based on behavioral and spontaneous resting-state brain activity, the present study demonstrated that people who acquired more information in the first learning trial were not necessarily faster learners in subsequent trials, at least in healthy older adults or those with mild cognitive impairment in China.

At the behavioral level, correlation and interaction analysis revealed that memory span and learning rate in older adults were independent. In PALT, the correlation between memory span and learning rate was not significant regardless of the number of healthy older adults $(707,69$, or 51$)$. Although memory span was significantly correlated with learning rate in AVLT, the coefficient was negative, indicating an opposite pattern to that in younger adults (Zerr et al. 2018). This suggests that the relationship between memory span and learning rate in item memory (AVLT) is affected by aging in different ways to that in associative memory (PALT), although the underlying mechanisms should be explored in future research.

With the development of pathological aging, an independent relationship between memory span and learning rate was further suggested. In $\mathrm{MCl}$ patients, the correlation between memory span and learning rate was not significant in either PALT or AVLT. These results indicated that the ability for older adults to initially acquire information in the first trial was not related to the increments in learning in subsequent trials. We argue that these contradictory findings (younger adults vs. healthy older adults and $\mathrm{MCl}$ patients) can be explained by the fact that memory span and learning rate are disproportionately affected by age and $\mathrm{MCl}$. The functions of memory span and learning rate are co-variating in younger adults, therefore, people who acquire more information in the first trial are also quick learners in the following learning trials. However, in the elderly and $\mathrm{MCl}$ patients, memory span might be impaired or damaged, but learning rate remains, while younger people have the opposite situation. The different variants of memory span and learning rate with the aging and the disease state might make memory span and learning rate not relevant. These results revealed that memory span and learning rate might be independent functions of each other.

Interaction analysis revealed that memory capacity (memory span vs. learning rate) may be differentially affected by test type (associative memory vs. item memory, indexed respectively by PALT vs. AVLT). The 
significant interaction indicated that the difference between item memory and associative memory in memory span was larger than that in learning rate; that is, older adults learned less in the associative memory test when compared to the item memory test in the initial learning trial; however, they did not learn associations as inefficiently as items. These findings highlight the separation of memory span and learning rate. It should be noted that the negative correlation between memory span and learning rate in AVLT and the different interaction patterns between AVLT and PALT did not result from a potential ceiling effect. As demonstrated in the Results, when we removed participants who were at or near the score ceiling, the score pattern remained unchanged.

Additionally, using forward stepwise regression analysis, we explored the contributions of several cognitive abilities to memory span and learning rate. We found that information processing speed tended to contribute more to memory span, nevertheless, higher intelligence scores may involve more in learning rate. The results were consistent with those of Zerr et al. who also reported that these two capacities were positively correlated, suggesting that they were not fully independent. We argue that these contradictory findings result from the co-variation of memory span and learning rate in younger adults. According to our results in the study, memory span and learning rate are disproportionately affected by age; thus, these two capacities were dissociable in older adults.

It is arguable that the influence of age and education should be controlled as covariates to exclude the possible impact in correlation analyses between indexes of brain activity and behavioral performance (memory span and learning rate) (Yin et al. 2015; Zheng et al. 2017). We believed that this argument not in line with previous research (Suri et al. 2017; Zerr et al. 2018), given that the main hypothesis here was whether memory span and learning rate were dissociable when disproportionately affected by certain factors. Age and education may be two important factors. Thus, if we considered them covariates, the dissociation between memory span and learning rate may not have been observed. In addition, we did not find a significant correlation between demographic characteristics (age, education) and differences in memory span and learning rate (standard score of memory span minus learning rate).

At the level of neural activity, the present study provided some evidence for the separation of memory span and learning rate. Based on spontaneous resting-state brain activity, we detected two features in the correlation analysis of behavioral performance and brain activity using three progressive indexes: single voxel level (ALFF), regional brain network level (ReHo), and whole-brain network level (DC). One was that the correlation coefficients were positive for learning rate, while negative for memory span in both PALT and AVLT. With regards to the distribution of brain regions, all regions associated with memory span were located in the right hemisphere, whereas most of the regions associated with learning rate were located in the left hemisphere (except one region in the left precuneus). These results imply that memory span and learning rate might be underpinned by different neural mechanisms.

Another important observation in network indexes level (ReHo and DC) was the significant associations between brain activity and learning rate in regions localized in the precuneus (especially the left 
precuneus), regardless of PALT or AVLT. In contrast, there was no association between the precuneus and memory span.

The ventral sub-region of the precuneus, perhaps the most connected hub in the cortex, is widely accepted as the central node of the default mode network (Gordon et al. 2014; Zhang \& Li 2012). It is a dynamic area of the brain, involved in a number of perceptual and cognitive abilities, such as episodic memory retrieval and metacognition, as well as integrating perceptions (Fornito et al. 2012). In addition, the precuneus is an important node in the attention circuit (Pereira-Pedro \& Bruner 2016), which is implicated in alertness and sustained attention (Andrews-Hanna et al. 2010; Boly et al. 2012).

It is expected that learning rate involved various learning processes including attention to information and metacognition to self-cognition memory retrieval to information Generally, higher ReHo and DC values indicate more efficient information processing and a more prominent role in the network (Zang et al. 2004; Zuo et al. 2012). In line with this, our results indicated that people with higher ReHo and DC values in the precuneus during resting-state showed higher learning speed. The brain activation mainly in the precuneus in our study is consistent with those of rs-fMRI studies on MCI PATIENTS (Pan et al. 2017; Yuan et al. 2016; Zhang et al. 2012).

The reasons underlying the selectivity of these effects for learning rate and not memory span remain unclear. One possible explanation is that, as an important component of the medial posterior parietal cortex, the dorsal sub-regions of the precuneus have substantial effects on episodic memory retrieval (Dörfel et al. 2009; Lundstrom et al. 2005; Lundstrom et al. 2003). Compared to memory span, learning rate relies more on the ability to retrieve information that has been remembered at previous trials as well as integrating new information into already existing memories. In line with this reasoning, the present study provides evidence that individual differences in resting-state precuneus function predict how quickly people learn across multiple trials.

Finally, with the development of pathological aging, our data suggest that the independent relationship between memory span and learning rate might be further confirmed on $\mathrm{MCl}$ patients. None of the correlation between memory span and learning rate was significant in either PALT or AVLT. These results indicate that the ability for older adults to initially acquire information in the first trial might not be related to the increments in learning in subsequent trials.

Mild cognitive impairment with memory complaints and deficits (amnestic mild cognitive impairment) is consistently shown to have the separation pattern in different cognitive processes (Mayes et al. 2019; Wang et al. 2013). For example, Wang et al. found the separating recollection and familiarity in emotional memory (Wang et al. 2013). Recently, a study of memory span and learning rate in amyotrophic lateral sclerosis patients have shown that ALS patients had lower scores on memory span than healthy participants, however, no significant differences in learning rate (Barulli et al. 2019). This results also demonstrated that memory span and learning rate might be independent with each other. 


\section{Conclusions}

This study could provide some novel evidence for the dissociation between memory span and learning rate at the behavioral and brain activity levels. This distinction is important, as more sensitive and efficient measures can be designed to detect cognitive deficits by separating memory span and learning rate if these capacities are disproportionately affected by disease. Similar to previous findings, tests with multiple learning trials (learning rate) are more sensitive for identifying older adults with $\mathrm{MCl}$ when compared to a single trial learning test ( $\mathrm{Li}$ et al. 2013). In addition, for cognitive interventions to agerelated mnemonic decline, it will be beneficial to differentially classify impairments in memory span and learning rate to design individual-based intervention strategies.

\section{Declarations}

\section{Ethics approval and consent to participate}

The present study was approved by the research ethics committee of the Institute of Psychology, Chinese Academy of Sciences (H11036). Written informed consent was obtained from each participant.

\section{Consent for publication}

The corresponding author, on behalf all authors, allows the publication of this Manuscript.

\section{Availability of data and materials}

The generated datasets are on-line open, DOI: 10.5281/zenodo.2583322

https://doi.org/10.5281/zenodo.2583322

\section{Competing interests}

The authors declare that they have no conflict of interest.

\section{Funding}

This work was supported by the National Key Research and Development Program of China (2017YFB1401203, 2018YFC2000303, 2018YFC2001701, and 2016YFC1305900), Beijing Municipal Science \& Technology Commission (Z171100000117006, Z171100008217006), International cooperation project of the Chinese Academy of Sciences (153111KYSB20180024), and National Natural Science Foundation of China $(31671157,31711530157,31470998,31861133011,31400895$, and 31871123).

\section{Authors' contributions}


PYW and SYC conceived the idea. PYW and JL supervised all aspects of the study including behavioral and fMRI data processing and analysis and drafted the manuscript. SYC conducted all the data analysis and drafted the manuscript, created all tables and figures. RL assisted in data analysis and revised the manuscript.

\section{Acknowledgements}

Not applicable

\section{References}

Almond NM, Morrison CM, and Moulin CJ. 2013. Episodic intertrial learning of younger and older adults: effects of word frequency. Neuropsychol Dev Cogn B Aging Neuropsychol Cogn 20:174-194. 10.1080/13825585.2012.679914

Anderson ND, Ebert PL, Jennings JM, Grady CL, Cabeza R, and Graham SJ. 2008. Recollection- and familiarity-based memory in healthy aging and amnestic mild cognitive impairment. Neuropsychology 22:177-187.

Andrews-Hanna JR, Reidler JS, Sepulcre J, Poulin R, and Buckner RL. 2010. Functional-anatomic fractionation of the brain's default network. Neuron 65:550-562.

Barulli MR, Piccininni M, Di Dio C, Musarò C, Grasso A, Tursi M, lurillo A, Lozupone M, Capozzo R, and Tortelli R. 2019. Episodic Memory and Learning Rates in Amyotrophic Lateral Sclerosis Without Dementia. Cortex.

Boly M, Massimini M, Garrido MI, Gosseries O, Noirhomme Q, Laureys S, and Soddu A. 2012. Brain connectivity in disorders of consciousness. Brain connectivity 2:1-10.

Davis HP, Small SA, Stern Y, Mayeux R, Feldstein SN, and Keller FR. 2003. Acquisition, recall, and forgetting of verbal information in long-term memory by young, middle-aged, and elderly individuals. Cortex; a journal devoted to the study of the nervous system and behavior 39:1063.

Dörfel D, Werner A, Schaefer M, Von KR, and Karl A. 2009. Distinct brain networks in recognition memory share a defined region in the precuneus. European Journal of Neuroscience 47:1947.

Dunlosky J, and Salthouse TA. 1996. A decomposition of age-related differences in multitrial free recall. Aging Neuropsychology:2-14.

Fornito A, Harrison BJ, Zalesky A, and Simons JS. 2012. Competitive and cooperative dynamics of largescale brain functional networks supporting recollection. Proceedings of the National Academy of Sciences 109:12788-12793. 
Gordon EM, Laumann TO, Adeyemo B, Huckins JF, Kelley WM, and Petersen SE. 2014. Generation and evaluation of a cortical area parcellation from resting-state correlations. Cerebral Cortex 26:288-303.

Guo Z, Liu X, Hou H, Wei F, Liu J, and Chen X. 2016. Abnormal degree centrality in Alzheimer's disease patients with depression: A resting-state functional magnetic resonance imaging study. Exp Gerontol 79:61-66. 10.1016/j.exger.2016.03.017

Hanseeuw B, Dricot L, Kavec M, Grandin C, Seron X, and Ivanoiu A. 2011. Associative encoding deficits in amnestic mild cognitive impairment: A volumetric and functional MRI study. Neuroimage 56:1743-1748.

Kramer JH, Yaffe K, Lengenfelder J, and Delis DC. 2003. Age and gender interactions on verbal memory performance. Journal of the International Neuropsychological Society Jins 9:97-102.

Lawton MP, and Brody EM. 1969. Assessment of older people: self-maintaining and instrumental activities of daily living. Gerontologist 9:179-186.

Lezak MD. 2004. Neuropsychological assessment. Oxford University Press, USA.

Li, Juan, Li, Huijie, Wang, and Pengyun. 2013. Differences in learning rates for item and associative memories between;amnestic mild cognitive impairment and healthy controls. Behavioral \& Brain Functions Bbf 9:29.

Li Z, Kadivar A, Pluta J, Dunlop J, and Wang Z. 2012. Test-retest stability analysis of resting brain activity revealed by blood oxygen level-dependent functional MRI. Journal of magnetic resonance imaging 36:344-354.

Liu H, Liu Z, Liang M, Hao Y, Tan L, Kuang F, Yi Y, Xu L, and Jiang T. 2013. Decreased regional homogeneity in schizophrenia: a resting state functional magnetic resonance imaging study. Addiction Biology 18:729-731.

Lundstrom BN, Ingvar M, and Petersson KM. 2005. The role of precuneus and left inferior frontal cortex during source memory episodic retrieval. Neuroimage 27:824.

Lundstrom BN, Petersson KM, Andersson J, Johansson M, Fransson P, and Ingvar M. 2003. Isolating the retrieval of imagined pictures during episodic memory: activation of the left precuneus and left prefrontal cortex. Neuroimage 20:1934-1943.

Machts J, Bittner V, Kasper E, Schuster C, Prudlo J, Abdulla S, Kollewe K, Petri S, Dengler R, and Heinze HJ. 2014. Memory deficits in amyotrophic lateral sclerosis are not exclusively caused by executive dysfunction: a comparative neuropsychological study of amnestic mild cognitive impairment. Bmc Neuroscience 15:83.

Maj M, Satz P, Janssen R, Zaudig M, Starace F, D'elia L, Sughondhabirom B, Mussa M, Naber D, and Ndetei D. 1994. WHO Neuropsychiatric AIDS Study, cross-sectional phase II: neuropsychological and 
neurological findings. Archives of General Psychiatry 51:51-61.

Mayes AR, Montaldi D, Roper A, Migo EM, Gholipour T, and Kafkas A. 2019. Amount, not strength of recollection, drives hippocampal activity: A problem for apparent word familiarity-related hippocampal activation. Hippocampus 29:46-59.

Moulin CJA, James N, Freeman JE, and Jones RW. 2004. Deficient Acquisition and Consolidation: Intertrial Free Recall Performance in Alzheimer's Disease and Mild Cognitive Impairment. Journal of Clinical \& Experimental Neuropsychology 26:1-10.

Pan P, Zhu L, Yu T, Shi H, Zhang B, Qin R, Zhu X, Qian L, Zhao H, Zhou H, and Xu Y. 2017. Aberrant spontaneous low-frequency brain activity in amnestic mild cognitive impairment: A meta-analysis of resting-state fMRI studies. Ageing Res Rev 35:12-21. 10.1016/j.arr.2016.12.001

Pereira-Pedro AS, and Bruner E. 2016. Sulcal pattern, extension, and morphology of the precuneus in adult humans. Annals of Anatomy-Anatomischer Anzeiger 208:85-93.

Petersen RC. 2004. Mild cognitive impairment as a diagnostic entity. Journal of internal medicine 256:183-194.

Qi R, Zhang L, Wu S, Zhong J, Zhang Z, Zhong Y, Ni L, Zhang Z, Li K, Jiao Q, Wu X, Fan X, Liu Y, and Lu G. 2012. Altered resting-state brain activity at functional MR imaging during the progression of hepatic encephalopathy. Radiology 264:187-195. 10.1148/radiol.12111429

Ranganath C. 2015. Binding Items and Contexts: The Cognitive Neuroscience of Episodic Memory. Current Directions in Psychological Science 19:131-137.

Seidman LJ. 1998. Neuropsychological testing. The Harvard mental health letter 14:4.

Serra L, Bozzali M, Cercignani M, Perri R, Fadda L, Caltagirone C, and Carlesimo GA. 2010. Recollection and familiarity in amnesic mild cognitive impairment. Neuropsychology 24:316-326.

Stella K, Troyer AK, and Rich JB. 2009. Prospective memory in amnestic mild cognitive impairment. Neuropsychology 15:407-415.

Suri S, Topiwala A, Filippini N, Zsoldos E, Mahmood A, Sexton CE, Singhmanoux A, Kivimäki M, Mackay $\mathrm{CE}$, and Smith S. 2017. Distinct resting-state functional connections associated with episodic and visuospatial memory in older adults. Neuroimage 159:122.

Wang P, Li J, Li H, Li B, Jiang Y, Bao F, and Zhang S. 2013. Is emotional memory enhancement preserved in amnestic mild cognitive impairment? Evidence from separating recollection and familiarity.

Neuropsychology 27:691. 
Wang X, Jiao D, Zhang X, and Lin X. 2017. Altered degree centrality in childhood absence epilepsy: A resting-state fMRI study. J Neuro/ Sci 373:274-279. 10.1016/j.jns.2016.12.054

Wechsler D. 1984. WMS-R: Wechsler memory scale-revised: manual: Psychological Corporation.

Westerberg CE, Paller KA, Weintraub S, Mesulam MM, Holdstock JS, Mayes AR, and Reber PJ. 2006. When memory does not fail: familiarity-based recognition in mild cognitive impairment and Alzheimer's disease. Neuropsychology 20:193-205.

Wolk DA, Signoff ED, and Dekosky ST. 2008. Recollection and familiarity in amnestic mild cognitive impairment: a global decline in recognition memory. Neuropsychologia 46:1965-1978.

Xu S, and Wu Z. 1986. The construction of "The Clinical Memory Test.". Acta Psychologica Sinica 18(1):100-108.

Yan C-G, Wang X-D, Zuo X-N, and Zang Y-F. 2016a. DPABI: data processing \& analysis for (resting-state) brain imaging. Neuroinformatics 14:339-351.

Yan CG, Wang XD, Zuo XN, and Zang YF. 2016b. DPABI: Data Processing \& Analysis for (Resting-State) Brain Imaging. Neuroinformatics 14:339-351. 10.1007/s12021-016-9299-4

Yin S, Zhu X, He R, Li R, and Li J. 2015. Spontaneous activity in the precuneus predicts individual differences in verbal fluency in cognitively normal elderly. Neuropsychology 29:961-970.

Yonelinas AP. 2002. The Nature of Recollection and Familiarity: A Review of 30 Years of Research $\nabla$. Journal of Memory \& Language 46:441-517.

Yong H, Liang W, Yufeng Z, Lixia T, Xinqing Z, Kuncheng L, and Tianzi J. 2007. Regional coherence changes in the early stages of Alzheimer's disease: a combined structural and resting-state functional MRI study. Neuroimage 35:488-500.

Yong $\mathrm{H}$, Zang $\mathrm{Y}$, Jiang T, Meng L, and Gong G. 2004. Detecting Functional Connectivity of the Cerebellum Using Low Frequency Fluctuations (LFFs).

Yu-Feng Z, Yong H, Chao-Zhe Z, Qing-Jiu C, Man-Qiu S, Meng L, Li-Xia T, Tian-Zi J, and Yu-Feng W. 2007. Altered baseline brain activity in children with ADHD revealed by resting-state functional MRI. Brain \& Development.

Yu J, Li J, and Huang X. 2012. The Beijing version of the Montreal Cognitive Assessment as a brief screening tool for mild cognitive impairment: a community-based study. BMC Psychiatry 12:156. 10.1186/1471-244x-12-156

Yuan X, Han Y, Wei Y, Xia M, Sheng C, Jia J, and He Y. 2016. Regional homogeneity changes in amnestic mild cognitive impairment patients. Neurosci Lett 629:1-8. 10.1016/j.neulet.2016.06.047 
Zang Y, Jiang T, Lu Y, He Y, and Tian L. 2004. Regional homogeneity approach to fMRI data analysis. Neuroimage 22:394.

Zerr CL, Berg JJ, Nelson SM, Fishell AK, Savalia NK, and McDermott KB. 2018. Learning Efficiency: Identifying Individual Differences in Learning Rate and Retention in Healthy Adults. Psychol Sci 29:14361450. $10.1177 / 0956797618772540$

Zhang S, and Li CR. 2012. Functional connectivity mapping of the human precuneus by resting state fMRI. Neuroimage 59:3548-3562.

Zhang Z, Liu Y, Jiang T, Zhou B, An N, Dai H, Wang P, Niu Y, Wang L, and Zhang X. 2012. Altered spontaneous activity in Alzheimer's disease and mild cognitive impairment revealed by Regional Homogeneity. Neuroimage 59:1429-1440. 10.1016/j.neuroimage.2011.08.049

Zheng Z, Li R, Xiao F, He R, Zhang S, and Li J. 2017. Sex Matters: Hippocampal Volume Predicts Individual Differences in Associative Memory in Cognitively Normal Older Women but Not Men. Frontiers in Human Neuroscience 11:93.

Zhou Y, Wang Y, Rao LL, Liang ZY, Chen XP, Zheng D, Tan C, Tian ZQ, Wang CH, and Bai YQ. 2014. Disrutpted resting-state functional architecture of the brain after 45 -day simulated microgravity. Frontiers in Behavioral Neuroscience 8:200.

Zuo XN, Di Martino A, Kelly C, Shehzad ZE, Gee DG, Klein DF, Castellanos FX, Biswal BB, and Milham MP. 2010. The oscillating brain: complex and reliable. Neuroimage 49:1432-1445.

10.1016/j.neuroimage.2009.09.037

Zuo XN, Ehmke R, Mennes M, Imperati D, Castellanos FX, Sporns O, and Milham MP. 2012. Network Centrality in the Human Functional Connectome. Cerebral Cortex 22:1862.

\section{Figures}



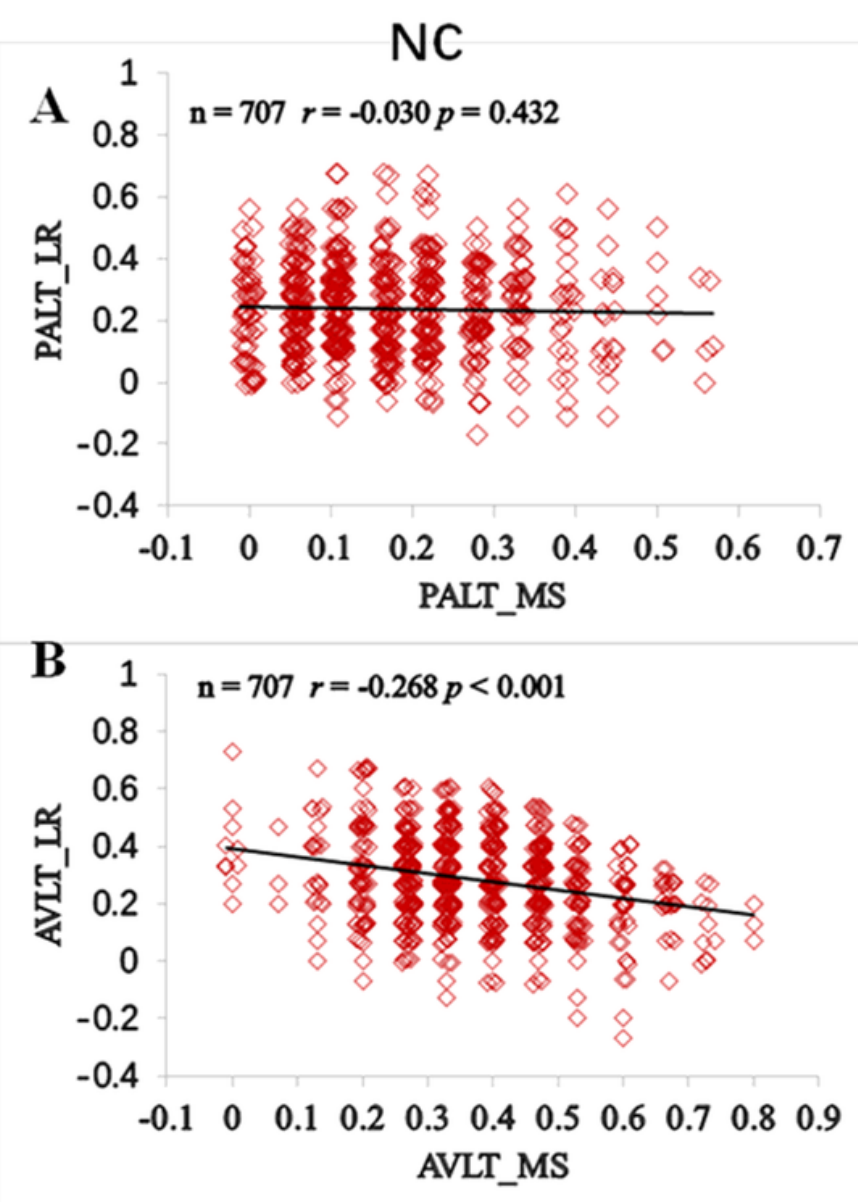

Figure 1

(figure captions not included)

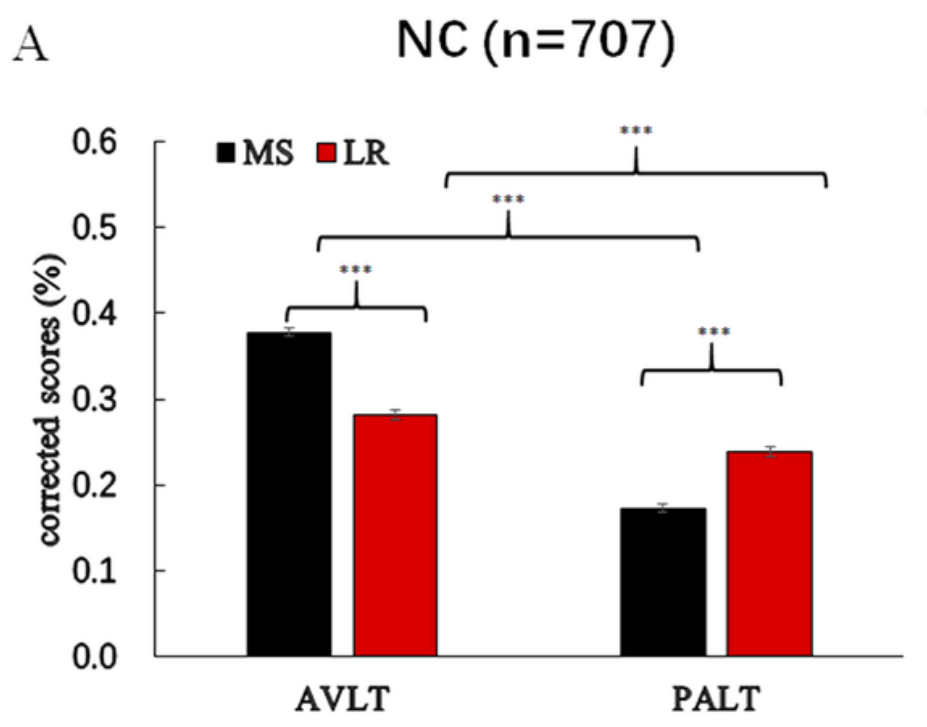

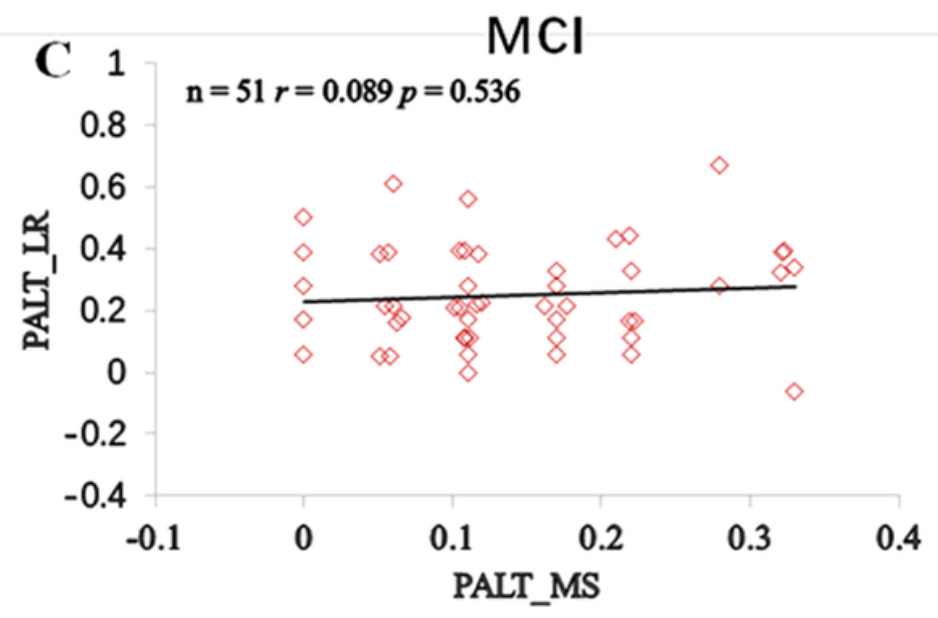

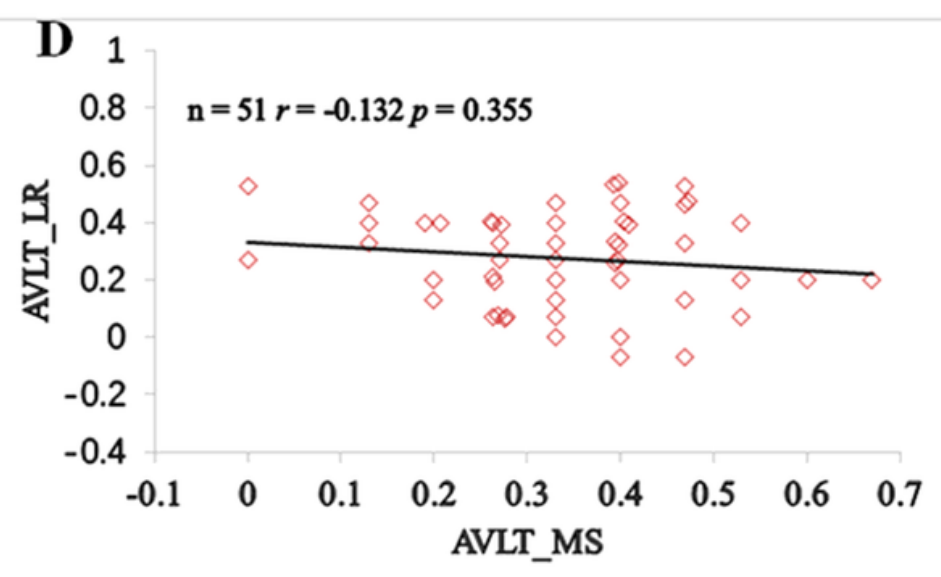

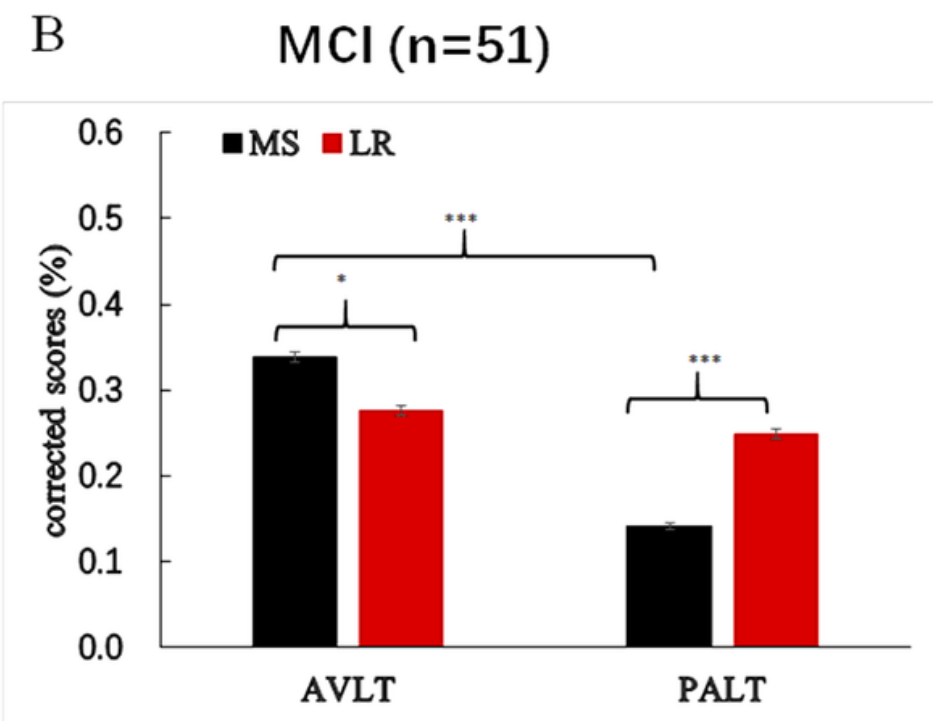

Figure 2 
(figure captions not included)
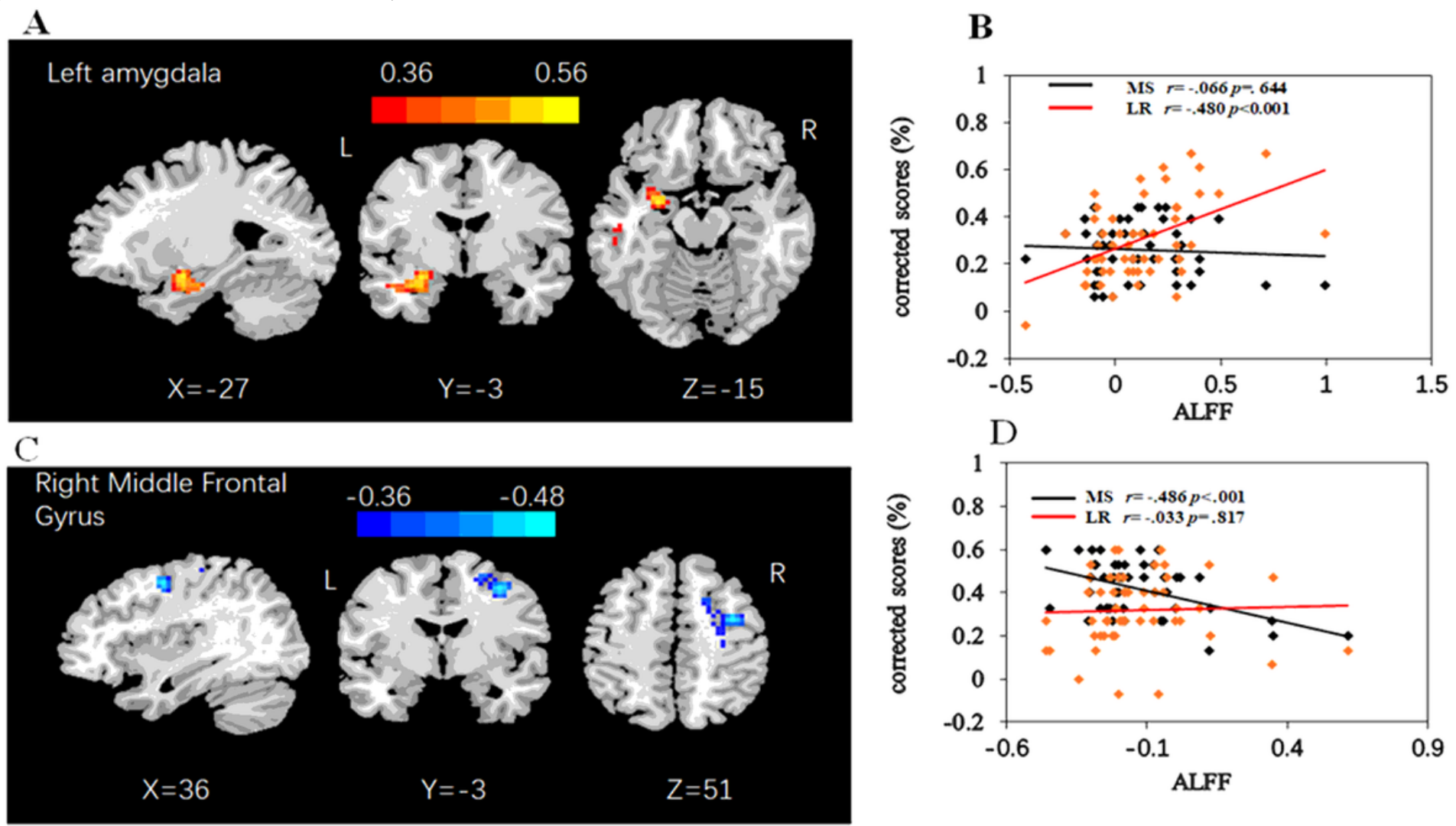

Figure 3

(figure captions not included) 


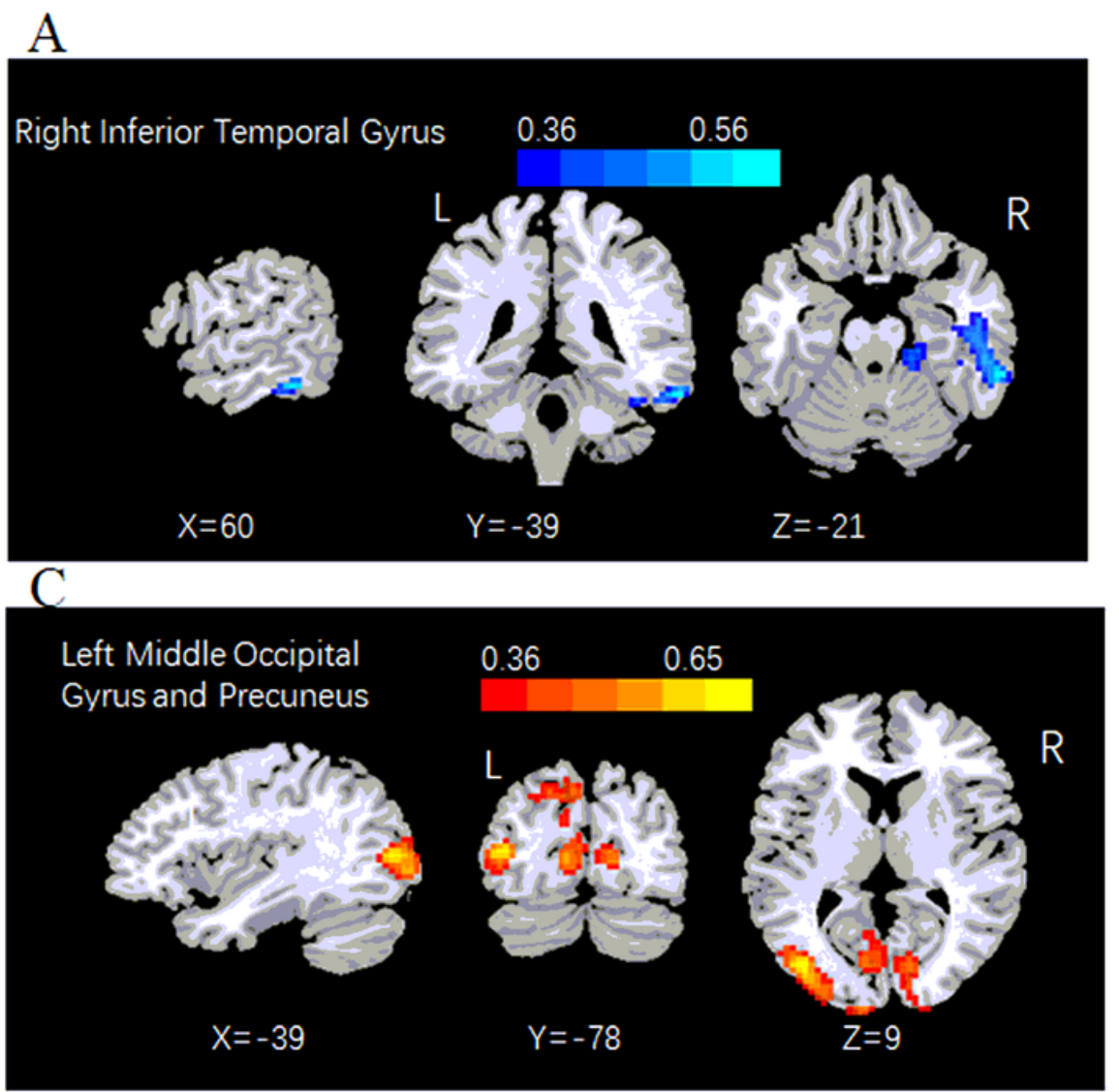

E

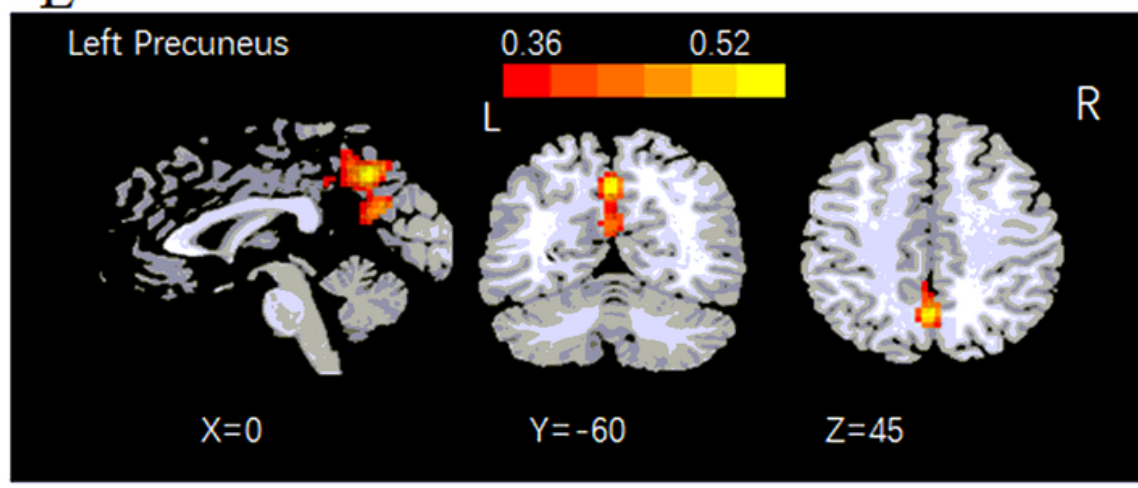

B
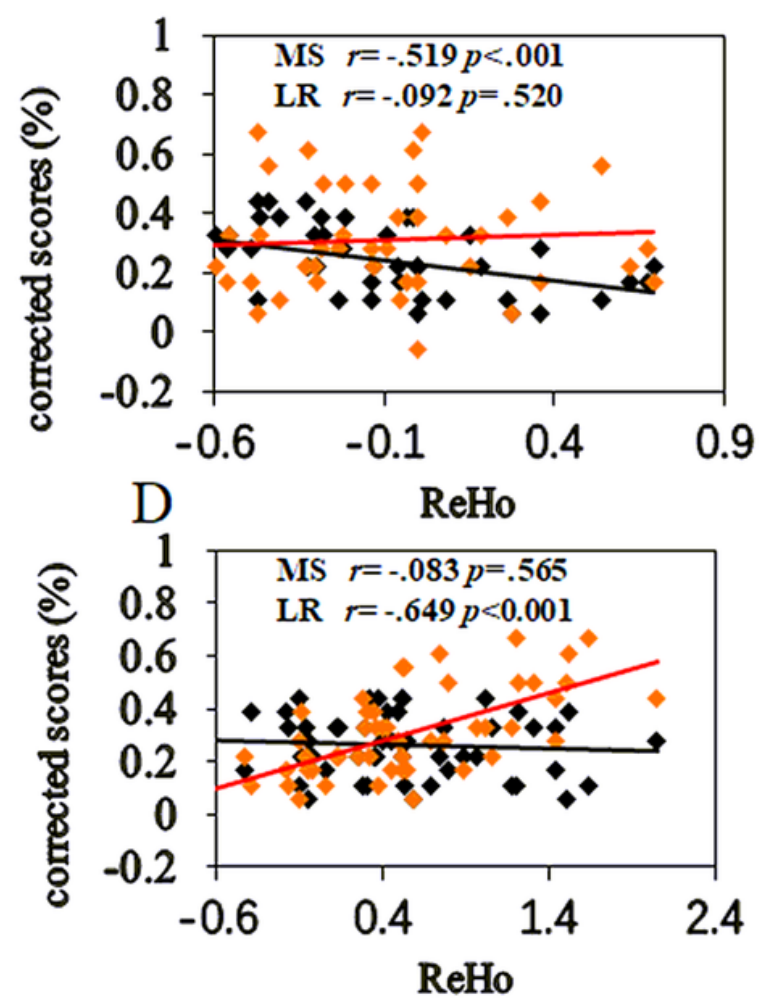

F

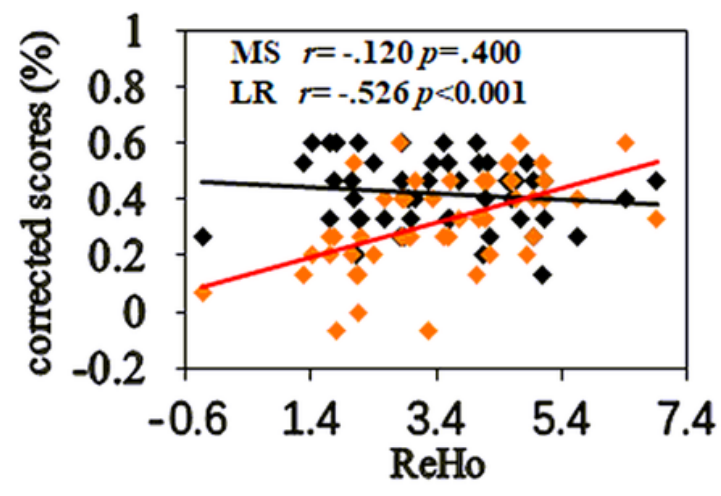

Figure 4

(figure captions not included) 

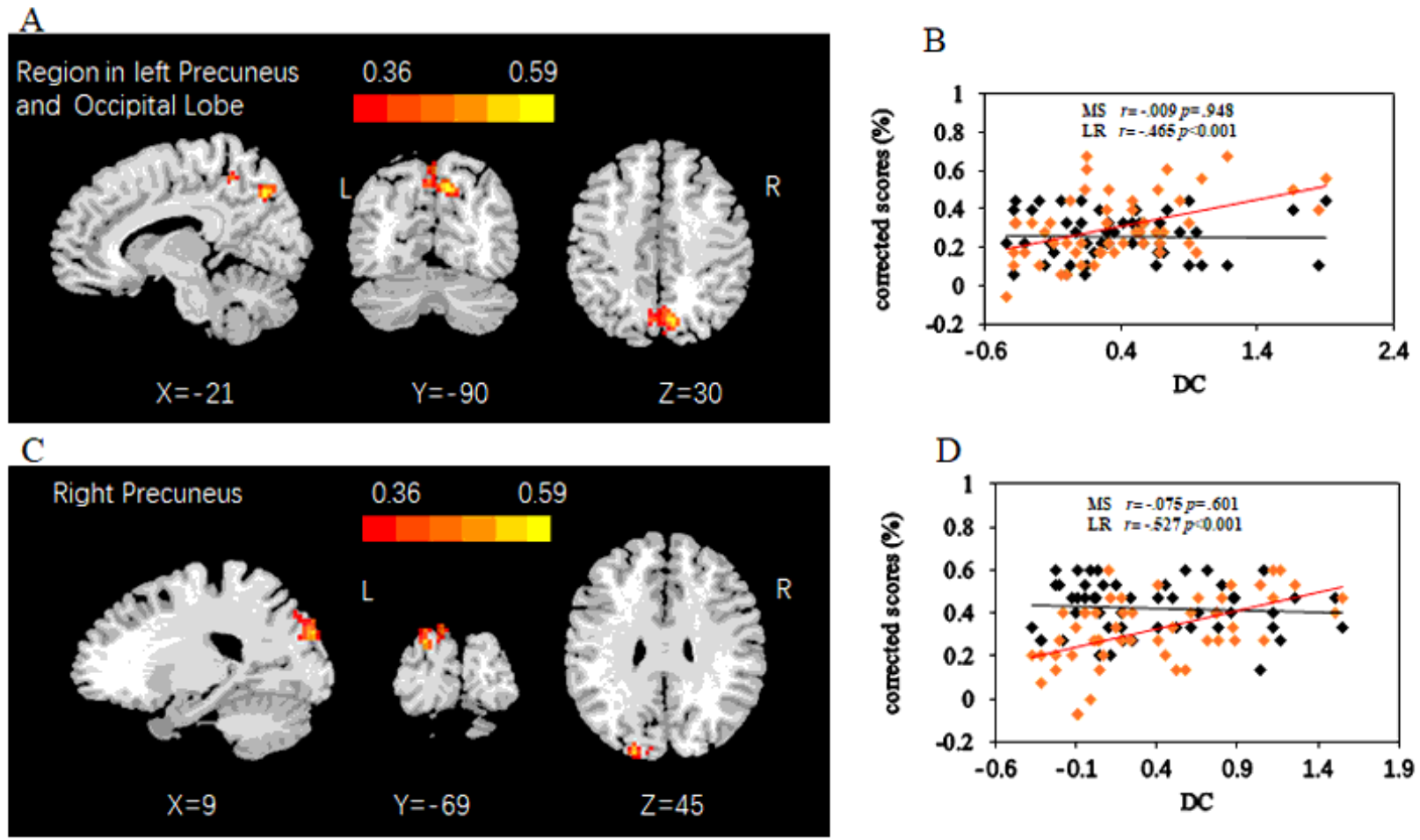

Figure 5

(figure captions not included)

\section{Supplementary Files}

This is a list of supplementary files associated with this preprint. Click to download.

- supplementarymaterials.docx 